\title{
Demonstration of a New Technique for the Transfer Printing of Graphene on Photonic Devices
}

\author{
Leili Abdollahi Shiramin', Alexander Bazin', Steven Verstuyft ${ }^{1}$, Sylvia Lycke², Peter Vandenabeele ${ }^{2}$, Gunther \\ Roelkens ${ }^{1}$, Dries Van Thourhout ${ }^{1}$ \\ ${ }^{1}$ Photonics Research Group, Department of Information Technology, Ghent University-IMEC, Technologiepark-Zwijnaarde 15, Ghent 9052, \\ Belgium \\ ${ }^{2}$ Raman Spectroscopy Group, Department of Archaeology, Ghent University, Sint-Pietersnieuwstraat 35, S-12, B-9000 Ghent, Belgium \\ E-mail address: leili.abdollahishiramin@ugent.be
}

\begin{abstract}
We demonstrate an automated method for transfer printing of micron sized graphene patterns on predefined sites on a photonic chip. Silicon nitride waveguides with graphene transferred on top exhibit an absorption loss of $0.054 \mathrm{~dB} / \mu \mathrm{m}$, in line with simulation results.

OCIS codes: 130.3130 Integrated optics materials; 310.3840 Materials and process characterization
\end{abstract}

\section{Introduction}

Graphene is usually grown on metal or dielectric substrates [1, 2], not well suited for optical applications. In order to exploit the promising properties of graphene in photonic integrated circuits a suitable transfer method should be developed. Several methods for the transfer of large scale graphene films have already been demonstrated [3, 4], but limited work on the transfer of micron sized graphene films has been carried out and all proposed methods are derived from the manual wet transfer method [5, 6].

Here, we present an automated dry method to transfer graphene pieces (called graphene coupons) on a specified device in the target sample. In our approach, first the graphene on the source substrate $\left(300 \mathrm{~nm} \mathrm{SiO}_{2}\right.$ on $\left.\mathrm{Si}_{\text {substrate }}\right)$ is patterned. Then a trench is etched in the $\mathrm{SiO}_{2}$ layer, $10 \mu \mathrm{m}$ away from the graphene patterns. Subsequently, a photoresist mask (Ti35E) protecting the graphene coupons and forming tethers towards the silicon substrate is defined. (Figure1a, b show respectively a top and side view). Then the sample is dipped in a buffered oxide etch to under-etch the $300 \mathrm{~nm} \mathrm{SiO}$ layer and release the graphene coupons with the resist on top. The prepared source sample and the target sample are then loaded on their dedicated stages in a transfer printer (X-celeprint, $\mu T P-100)$. Figure 1c shows the transfer printer including the source and target stages for the source and target samples and a cleaning pad. The transfer is done using a PDMS stamp attached to a glass plate loaded in the stamp holder. This stamp holder has the capability of moving accurately above the stages.

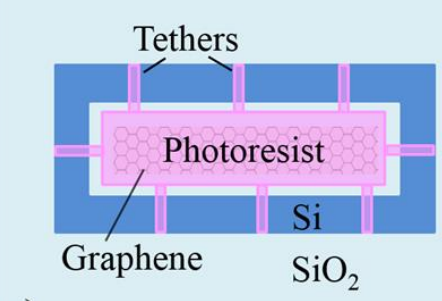

a)

Fig. 1. The schematic of a graphene coupon on the source sample. a) Top view. b) Side view. c) The transfer printer machine showing the source, target, and cleaning pads, the glass plate with the PDMS stamp (not visible) attached to the stamp holder.

The transfer is based on controlling the adhesion between the stamp, the resist covered graphene coupon and the source and target samples in respectively the pickup and printing steps. In the former, the graphene coupons are picked up with high speed while in the latter they are printed at slow speed to leave the graphene attached to the target substrate.

\section{Graphene transferred to Silicon Nitride waveguide}

To investigate the quality of the transferred graphene, a series of graphene coupons of size $10 \times 250 \mu \mathrm{m}^{2}$ were transferred on planarized SiN waveguides. Then the resist was removed and the graphene layers were patterned to realize waveguides with different length of graphene on top. Figure $2 \mathrm{a}$ is an image from a patterned graphene coupon on a waveguide. The waveguide absorption as function of the length of the graphene layer is depicted in Figure $2 \mathrm{~b}$. As expected the loss increases linearly with graphene length allowing us to extract an absorption loss of $0.054 \mathrm{~dB} / \mu \mathrm{m}$, in line with simulations [7]. A Raman measurement on the transferred graphene and the comparison with a graphene reference sample show the graphene quality is preserved after the transfer (Figure2c). 

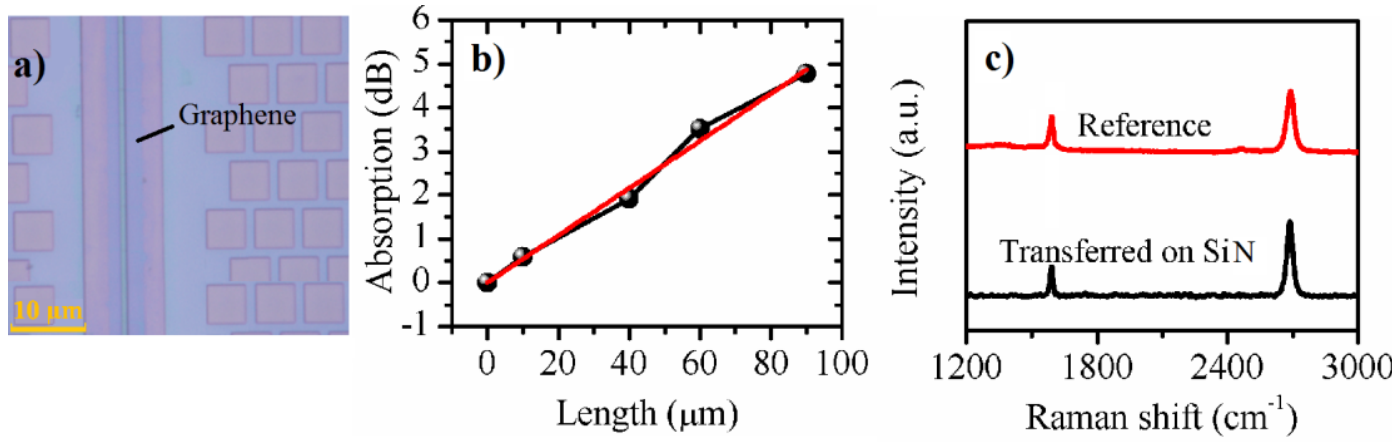

Fig. 2. a) Transferred graphene on the SiN waveguide. b) Graphene absorption versus graphene length. c) Raman measurement on the transferred graphene on the SiN waveguide in comparison with reference graphene.

\section{Graphene resistivity characterization}

In a second experiment we transferred graphene coupons on Palladium (Pd) electrode patterns defined on a $\mathrm{SiO}_{2}$ sample using a standard lift-off process and extracted the graphene contact and sheet resistance. After resist patterning but before metal deposition the $\mathrm{SiO}_{2}$ was slightly etched, so that after lift-off the sample was fully planarized. The graphene coupons were transferred on the Pd contacts using the transfer printing method outlined above and the photoresist was removed. Figure $3 \mathrm{a}$ is an image from a patterned graphene coupon on the Pd contacts. The size of the graphene layers on all of the Pd patterns is $4 \times 25 \mu \mathrm{m}^{2}$. The contact pads separation is varied from 3 to $25 \mu \mathrm{m}$. From Figure 3b, a contact resistance of $2990 \Omega . \mu \mathrm{m}$ and sheet resistance of $398 \Omega / \mathrm{sq}$ is extracted, reasonable values for CVD graphene [8].
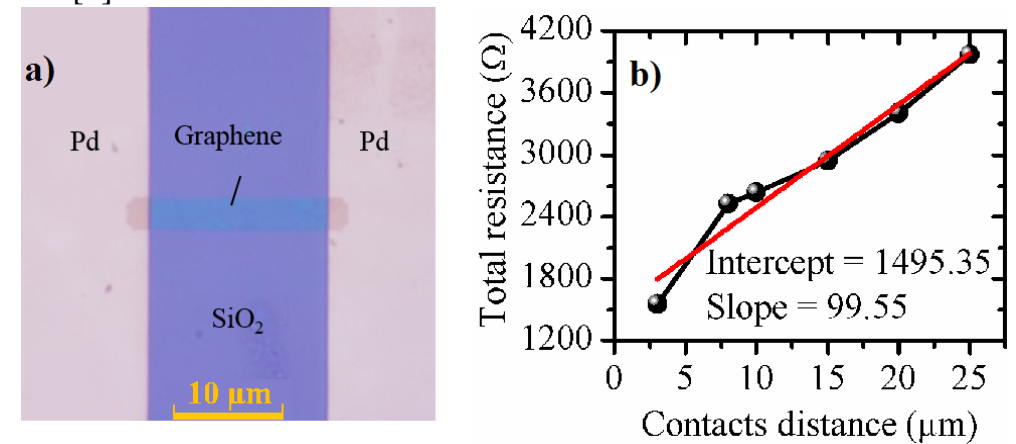

Fig. 3. a) Graphene on the pre-patterned palladium contacts. b) Total resistance as function of palladium contact's distance

\section{Conclusion}

In summary, we demonstrated an automated transfer method for patterned graphene films on any device of interest. The presented approach paves the way for the wafer scale integration of graphene and optoelectronic devices.

\section{Acknowledgement}

We acknowledge support by the EU commission through the Graphene Flagship Project and by the Ghent University BOF fund. Part of the work was supported by the H2020 project TOPHIT under the ICT2-2014 Smart System Integration program.

\section{References}

[1] H. Wang, et al, "Direct CVD Graphene Growth on Semiconductors and Dielectrics for Transfer-Free Device Fabrication," Adv. Mater 28, 4956-4975 (2016).

[2] Y. Kim, et al, "Direct growth of patterned graphene on $\mathrm{SiO}_{2}$ substrates without the use of catalysts or lithography," Nanoscale 6, 10100-10105 (2014).

[3] S. Bae, et al, "Roll-to-roll production of 30-inch graphene films for transparent electrodes," Nat. Nanotech 5, 574-578 (2010).

[4] A. Bosca, et al, "Automatic graphene transfer system for improved material quality and efficiency" Sci. Rep 6 (2016).

[5] A. Reina, et al, "Transferring and Identification of Single- and Few- Layer Graphene on Arbitrary Substrates," Phys. Chem C 112, 1774117744 (2008).

[6] Y. Bie, et al, "Site-Specific Transfer-Printing of Individual Graphene Microscale Patterns to Arbitrary Surfaces," Adv. Mater 23, 3938-3943 (2011).

[7] L. Abdollahi Shiramin, et al, "Graphene Modulators and Switches Integrated on Silicon and Silicon Nitride Waveguide," JSTQE 23, (2017).

[8] K.Asadi, et al,“Up-Scaling Graphene Electronics by Reproducible Metal-Graphene Contacts,” ACS Appl. Mater. Interfaces 7, 9429 (2015). 